\title{
A Programmable Transceiver Structure of Multi-rate OFDM-CDMA for Wireless Multimedia Communications
}

\author{
Po-Wei Fu \\ Department of Electronical Engineering and \\ Graduate Institute of Communication Engineering \\ National Taiwan University, Taipei, Taiwan, R.O.C. \\ powei@santos.ee.ntu.edu.tw
}

\author{
Kwang-Cheng Chen \\ Department of Electrical Engineering and \\ Graduate Institute of Communication Engineering \\ National Taiwan University, Taipei, Taiwan, R.O.C. \\ chenkc@cc.ee.ntu.edu.tw
}

\begin{abstract}
Tivo multi-rate transmission schemes, multi-code (MC) and variable-spreading-length (VSL) code, for realizing multimedia communications on three types of OFDM-CDMA systems are proposed. We integrate them into a programmable structure such that the operation can be controlled and adjusted by system parameters and thus the transceiver can be used in different systems without changing the fundamental hardware and software architecture, which serves the trend of software-radio for future application.
\end{abstract}

\section{Introduction}

Multimedia communication will be the main strean in the future communication services and it introduces challenges in effective transmission. As code-division multiple-access (CDMA) being utilized for the third generation and future communication systems, it is an important subject to realize multimedia services based on CDMA. Based on previous research, Multi-Code (MC) and Various-Spreading-Length (VSL) access schemes are the two most fundamental and widely applied multi-rate schemes [1]. On the other hand, Orthogonal-Frequency-Division-Multiplexing (OFDM) has been widely used in high-speed digital communications, which can be implemented efficiently by the digital technique of Fast-Fourier-Transform (FFT)[2] and known as a solution to combat the problem of the highly hostile mobile channels in high-speed transmissions. Furthermore, combining CDMA and OFDM results in finer partition of radio resource, which makes the resource allocation more effective. Therefore, OFDM-CDMA for multimedia applications is an attractive candidate for the $4^{\text {th }}$ generation wireless communication systems and its realization by a flexible software-defined architecture is of significant interests. Recent years, multiple access schemes based on the combination of CDMA and OFDM have been proposed, and they can be generally divided into three types, namely $\mathrm{MC}$ CDMA, MC-DS-CDMA, and MT-CDMA respectively [3]. To realize multi-data-rate transmission, we separately proposed two transmission methods, based on MC and VSL strategies, for the three OFDM-CDMA scenarios. Besides, we design a transceiver architecture accommodating these six multi-rate OFDM-CDMA

*This research is supported in part by Ministry of Education, Taiwan, R.O.C. and by the Integrated Programmable Communications, Inc. scenarios and show its programmability such that system operation can be controlled and reconfigured by adjusting parameters in software. It supports the accommodation in different systems with only one fundamental hardware and software architecture.

\section{Multi-Rate OFDM-CDMA Systems}

Assume there is a basic data rate supported in systems and the data rate of each user is an integer multiple $m$ of the basic data rate, said a user with rate $m$. The detailed structures of the multirate (MR)-OFDM-CDMA systems are described in the following. In transmitting aspects,

\section{(1) Multi-rate $M C-C D M A$}

a. MC access

The data stream of a user with rate $m$ is first multiplexed into $m$ different streams with basic rate and then each is treated as an individual (effective) user with individual spreading codes. After multiplexing, each stream is serial-to-parallel (S/P) converted to $P$ outputs, where the number of sub-carriers in the system depends on $P$. For frequency-domain spreading, symbols on each output is copied into $F$ branches, where $F$ is the constant spreading factor of the spread spectrum operation in the system and the signal at each sub-stream is then multiplied by the corresponding bit of the spreading codes. Thus, there are $P F$ parallel outputs of each effective user after the frequency domain spreading. After combining all the $P F$ parallel outputs from other users, they are transmitted by $P F$ orthogonal carriers correspondingly, where Inverse Discrete Fourier Transform (IDFT) can perform this modulation on orthogonal carriers equivalently. Label the effective users as user $1,2, \ldots$, and the transmitted BPSK signal of a system containing $M$ data rates in baseband is

$$
x(t)=\sum_{k=1}^{K} \sum_{j=1}^{P} A_{k} b_{k p} \sum_{f=1}^{F} c_{k f} e^{j 2 \pi f_{r f^{t}}}, 0 \leq t \leq P T_{s},
$$

where $K=\sum_{m=1}^{M} m K_{m}$ is the number of total effective users, $b_{k p}$ is the $p$ th symbol of the $k$ th effective user, and $A_{k}$ is the transmitted amplitude of the $k$ th effective user. $\Delta f \equiv f_{p, f+1}-f_{p, f}=1 / P T_{g}$, where $T_{s}$ is the symbol duration of the data stream with basic 
rate before S/P conversion:- $e_{i j} \in\{1\}$ denotes the $f$ th bit of the spreading codes used by the $k$ th effective user.

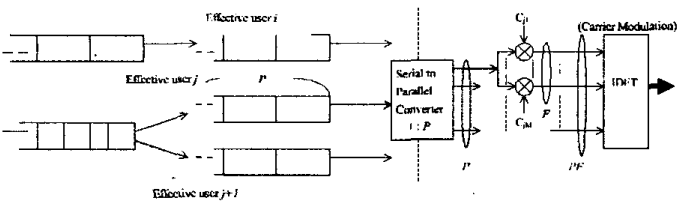

b. VSL access

The data stream from a user with rate $m$ is directly $S / P$ converted into $P m$ sub-streams, where the number of sub-carriers in the system is still $P F$ as in MC access. Symbols on each substream are copied into $F / m$ branches and then the symbols on each branch are multiplied by the corresponding bit of spreading codes. Note that $F / m$ should be chosen as an integer in our system design. Regardless any date rate, there are totally $P F$ parallel signal outputs after such frequency domain spreading. Combining all the $P F$ parallel signals from other users, they are transmitted by orthogonal carriers. The bandwidth of each sub-carrier and the overall occupied bandwidth are the same as in MC access. The transmitted signal in a VSL system containing $M$ data rates is

$$
x(t)=\sum_{m=1}^{M} \sum_{k=1}^{K} \sum_{p=1}^{m P} A_{m k} b_{m k p} \sum_{f}^{F / n z} c_{m k f} e^{j 22 \pi j^{\prime} t}, 0 \leq t \leq P T_{s},
$$

where $K_{m}$ denotes the number of users with rate $m, b_{m m p}$ is the $p$ th symbol of the $k$ th user with rate $m, A_{m k}$ is the transmitted amplitude, and $\Delta f=1 / P T_{s} \cdot c_{m k f} \in\{ \pm 1\}$ denotes the $f$ th bit of the spreading codes used by the $k$ th user with rate $m$.

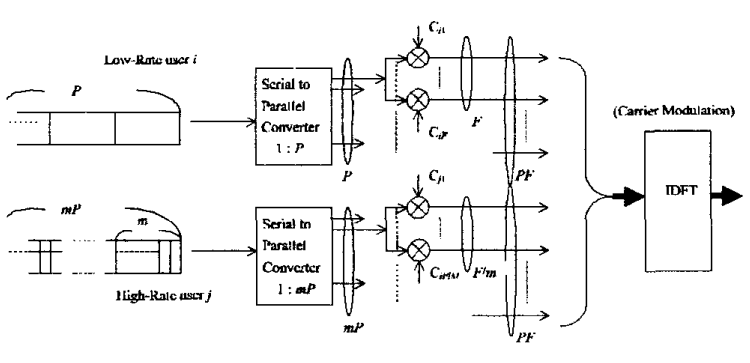

\section{(2) Multi-rate MC-DS-CDMA}

\section{a. $\mathrm{MC}$ access}

The data stream of rate $m$ is first multiplexed into $m$ different streams with basic rate and each is treated as an individual (effective) user with individual spreading codes. Each stream is then $\mathrm{S} / \mathrm{P}$ converted into $P$ parallel sub-streams, where $P$ is the number of sub-carriers in transmission. At the spectrum-spreading stage, the sub-streams from the same effective user are spread by the same spreading codes with facior $F$ via cyclically multiplying the codes. Combining all the $P$ parallel spread signals from other effective users, they are transmitted by orthogonal carriers correspondingly. Note that if the system adopts the strategy in [5] to expend the transmission diversity with a factor $L$, each substream before spreading stage should be copied into $L$ identical branches and these data-streams from the same effective user are then. spread .by the... same spreading.codes and transmitted via different sub-carriers. Thus, the number of sub-carriers in transmission is generally $P L$. The transmitted signal is,

$x(t)=\sum_{k=1}^{K} \sum_{p=1}^{P} A_{k} b_{k p} \sum_{f=1}^{F} c_{k j} \varphi\left(t-f T_{c}\right) \sum_{l=1}^{L} e^{j 2 \pi \pi_{j k^{t}}}, 0 \leq t \leq P T_{s}$,

where $K=\sum_{m=1}^{M} m K_{m}$ is the number of effective users, $\operatorname{and} \varphi(t)$ is the unit-rectangular function with duration $T_{c}$, and $\Delta f=F / P T_{s}$.

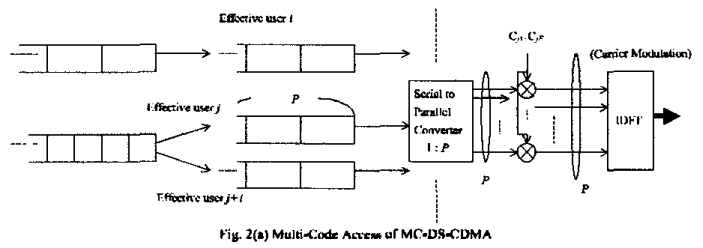

b. VSL access

Regardless any date rate, the data stream of each user is directly $\mathrm{S} / \mathbf{P}$ converted into $\boldsymbol{P}$ sub-streams. If the user is with rate $m$, each sub-stream is spread with factor $F / m$ by cyclically multiplying the same spreading codes. Combining all the $P$ parallel siguals form other users, they are transmitted via orthogonal carriers. Also note that if the transmission diversity is expended with a factor $L$, each sub-stream before spreading stage should be copied into $L$ identical branches and these data-streams from the same user are then spread by the same spreading codes and transmitted by different sub-carriers The number of subcarriers in transmission is still generally $P L$. The transmitted signal is

$$
x(t)=\sum_{i n=1}^{M} \sum_{k=1}^{K_{k}} \sum_{z=1}^{m} \sum_{p=1}^{P} A_{m k} b_{m k k s} \sum_{f}^{F / m} c_{m n k} \varphi\left(t-f T_{c}-\frac{(y-1) P T}{m}\right) \sum_{i=1}^{L} e^{j 2 \pi f_{f^{\prime}}},
$$
$0 \leq t \leq P T_{s}$

where $K_{1 m}$ is the number of users with rate $m$ and $\Delta f=F / P T_{s}$

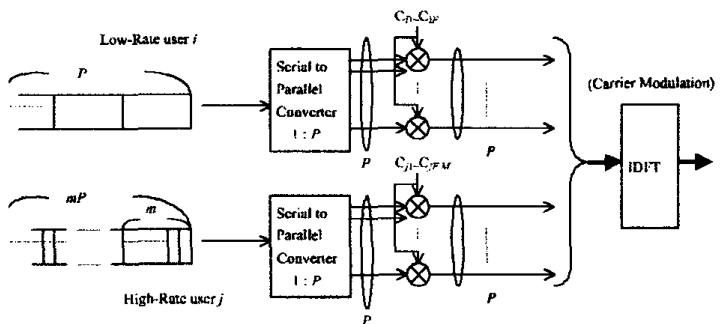

Fig. 2(h) Varizhle-Spreading-Lemgll] Access of MC-DS-CDMA

(3) Multi-rate MT-CDMA

a. $M C$ access

The data stream of rate $m$ is first multiplexed into $m$ different streams with basic rate and each is treated as an individual (effective) user with individual spreading codes. Each streams is then $\mathrm{S} / \mathrm{P}$ converted to $P$ outputs, where $P$ is the number of subcarriers in the system. The $P$ parallel outputs from the same effective user are spread with factor $F$ by identical spreading 
codes via cyclical multiplying the codes. Unlike in MC-DSCDMA, after combining all the $P$ spread signals from other users, they are transmitted by carriers whose implicit orthogonality exists corresponding to the signals before spreading. NOC-IDFT (see Appendix) performs this modulation equivalently instead of regular IDFT. The transmitted signal is

$$
x(t)=\sum_{k=1}^{K} \sum_{p=1}^{P} A_{k} b_{k p} \sum_{j=1}^{F} c_{k} \varphi\left(t-f T_{c}\right) e^{j 2 \pi f_{j},}, 0 \leq t \leq P T_{s},
$$

where $K=\sum_{m=1}^{M} m K_{m}$ is the number of total effective users and $\Delta f=1 / P T_{s}$.

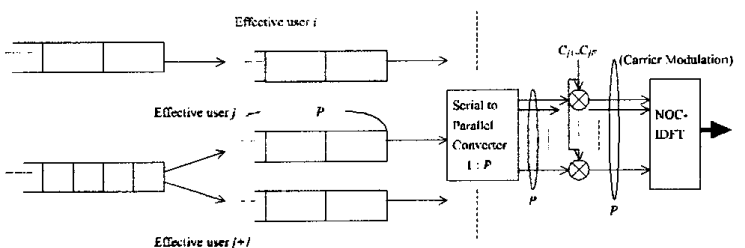

b. VSL access

The data stream from a user regardless any rate is $\mathrm{S} / \mathrm{P}$ converted into $P$ parallel sub-streams, where $P$ is the number of sub-carriers in the systems. Signal at each sub-stream is spread with factor $F / m$ by cyclical multiplying the spreading codes. After combining all the $P$ spread signals from other users, they are transmitted by carriers, where the implicit orthogonality exists between signals before spreading. The transmitted signal is

$$
\begin{aligned}
& x(t)=\sum_{m=1}^{M} \sum_{k=1}^{K_{m}} \sum_{g=1}^{m} \sum_{p=1}^{P} A_{m k} b_{m k \mathrm{~g} p} \sum_{f}^{F / m} c_{m k g} \varphi\left(t-f T_{c}-\frac{(g-1) P T_{s}}{m}\right) e^{j 2 \pi f_{p} t}, \\
& 0 \leq t \leq P T_{s},
\end{aligned}
$$

where $K_{i n}$ is the number of users with rate $m$ and $\Delta f=1 / P T_{s}$.

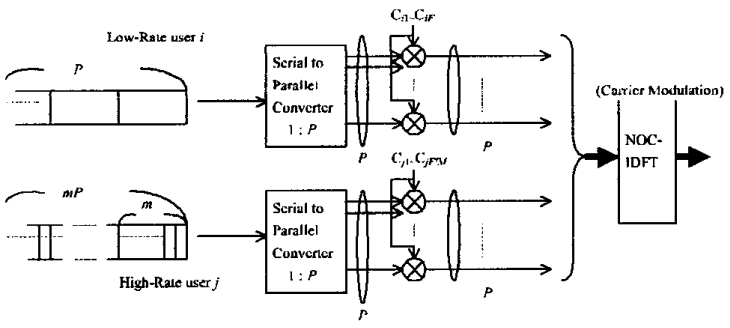

Fig. 3rb) Vurriable-Spreading-Length $\Lambda$ ccess of MT-COMA

In receiving aspect, a general structure is illustrated as Fig. 4. In general, the receiver performs inverse functions corresponding to those in the transmitter.

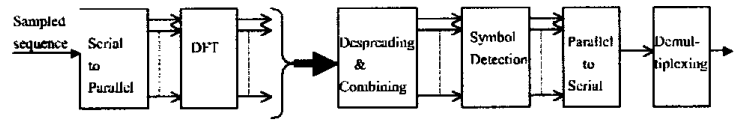

Fig. 4 The general receiving structure of MR-OFDM-CDMA

The detailed architectures and functions of different scenarios are described in the following sections for both transmitter and receiver in programmable design.

\section{Programmable Architecture}

\section{Programmable OFDM-CDMA transmitter.}

Two multi-rate access strategies and three OFDM-CDMA methods result in totally six multi-rate OFDM-CDMA scenarios. The proposed architecture of programmable transmitter is depicted in Fig. 5, which also shows the control functions, FI F6, that should be executed in operation. Each function can be adjusted by some parameters, which includes: choice of access scheme, choice of adopted OFDM-CDMA scheme, data rates, and the spreading factors of each data stream. These functions are defined as:

F1: It determines the number of enabled Main Branches (MB). It is executed on a multiplexer after receiving the data stream from a user. If the system is operated on multi-code access mode, it multiplexes the data stream into $m$ sub-streams by enabling $m$ branches. If it operates on VSL mode, just forward the data stream on one branch.

F2: It determines how many Paraltel Output Branches (POB) corresponding to the $\mathrm{S} / \mathrm{P}$ ratio will be enabled. Only under the scenario of VSL-MC-CDMA, POB is set to be $m P$; otherwise, POB is $P$.

F3: Function 3 determines the number of enabled Sub-Branches (SB), which is a copy of the former stream, of each POB and the type of spreading. If the scenario is MC-CDMA, the enabled SB is $F$ for multi-code access and $F / m$ for VSL access. If the scenario is MC-DS-CDMA and MT-CDMA, SB is $L$ and 1 respectively for both access methods. Cyclical multiplying by corresponding codes on each SB is set for MC-DS-CDMA and MT-CDMA. In MC-CDMA mode, signal on each SB is spread by multiplying one corresponding bit of spreading codes.

F4: To satisfy the required number of parallel inputs for IFFT operation, fuuction 4 pads zeros according to the selected scenario, which results in regular padding for regular IFFT and circularshift padding for NOC-IFFT. The circular period is $F$ in MC mode and $F / m$ in VSL mode. In example of radix-2 algorithm, the number of Padded Zeros (PZ) should be $2^{\left\lceil\log _{2} N_{\mathrm{z}}\right\rceil}-N_{b}$ in regular IFFT, where $N_{b}$ denotes the number of parallel inputs. For NOCIFFT operation in MT-CDMA modes, PZ should be $F\left(2^{\left\lceil\log _{2} N_{h}\right\rceil}-N_{b}\right)$ and allocated in circular-shift type.

F5: Function 5 decides the number of operation points (OP) in IFFT for each scenario. OP equals the number of outputs in F4. F6: Function 6 determines the enabled input branches of the P/S converter and the conversion ratio at different scenarios.

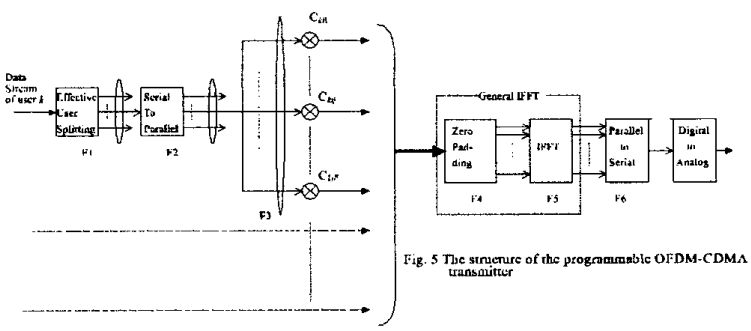


Table 1 summarizes the parameters of F1 F5 in different scenarios, the operation period in $\mathrm{F} 6$, and the resultant bandwidth. The involved notations are defined as:

MC: multi-code, VSL: Variable-Spreading-Length A: MC-CDMA, B: MC-DS-CDMA, C: MT-CDMA $m$ : data rate in unit of basic rate

$F$ : spreading factor of a basic data-rate user

$L$ : the diversity expansion of MC-DS-CDMA

CNS: constant bit multiplying, CYC: cyclical multiplying

\begin{tabular}{|c|c|c|c|c|c|c|c|}
\hline cnarivi & $\begin{array}{l}\mathrm{F} 1 \\
\mathrm{MB} \\
\end{array}$ & $\begin{array}{c}\mathrm{F} 2 \\
\mathrm{POB}\end{array}$ & $\begin{array}{l}\text { F3 } \\
\text { SB }\end{array}$ & $\begin{array}{l}4 \\
P Z\end{array}$ & $\begin{array}{l}\text { F5 } \\
\text { OP }\end{array}$ & $\begin{array}{c}\text { F6 } \\
\text { Period }\end{array}$ & Bandwidth" \\
\hline $\begin{array}{l}(A, m \\
M C)\end{array}$ & $m$ & $P$ & $F$; CNS & $\begin{array}{l}2^{[\log (f F)]_{a} P F} \\
\text { regular }\end{array}$ & $2^{\operatorname{lig}_{2}(P F)}$ & $P T_{s}$ & $\frac{P k^{k}-1}{P T_{x}}+\frac{1}{T}$ \\
\hline $\begin{array}{l}(A, \mathrm{~m}, \\
V S L)\end{array}$ & 1 & $m P$ & $\begin{array}{l}F / m: \\
\mathrm{CNS}\end{array}$ & $\begin{array}{l}2^{\left[\log _{2} P F\right]}-P F \\
\text { regular }\end{array}$ & 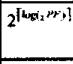 & $P T_{s}$ & $\frac{P F-1}{P T}+\frac{1}{r}$ \\
\hline $\begin{array}{c}\left(B, m_{1}\right. \\
M C, L)\end{array}$ & $m$ & $P$ & $L ; \mathrm{CYC}$ & $\begin{array}{c}2^{\left[\operatorname{los} x_{1} S L 1\right]}-P L \\
\text { regular }\end{array}$ & 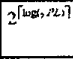 & $P_{T} / F$ & $\frac{(P I-1)}{P T_{1}}+\frac{1}{T}$ \\
\hline $\begin{array}{l}(B, m, \\
V S L, L)\end{array}$ & 1 & $P$ & $L ; \mathrm{CYC}$ & 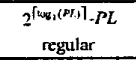 & $2^{\log _{2}(P 7 ., 1}$ & $P T, / F$ & $\frac{(P L-1)}{P T_{*}}+\frac{1}{T}$ \\
\hline $\begin{array}{c}(C, m \\
M O) \\
\end{array}$ & $m$ & $\bar{P}$ & $1 ; \mathrm{CYC}$ & $\begin{array}{l}F\left(2^{\log _{1} f}-P\right) \\
\text { circuiar-shift( }(F)\end{array}$ & $F 2^{\sqrt{\left.\operatorname{lom}_{51} p\right]}}$ & $P T_{\kappa} / F$ & $\frac{P-1}{P T_{1}}+\frac{1}{T}$ \\
\hline $\begin{array}{l}(C, m, \\
V S L)\end{array}$ & 1 & $P$ & $1 ; \mathrm{CYC}$ & $\begin{array}{c}F\left(2^{\log _{1} P}-P\right) \\
\text { circular-shift }(F / m)\end{array}$ & $F 2^{\left[b_{\left.b_{y}, p^{\prime}\right]}\right.}$ & $P T, F$ & $\frac{P-1}{P T_{1}}+\frac{1}{T}$ \\
\hline
\end{tabular}

The $T$ is defined in section 4 for introducing guard time at each scenario.

According to the scenario selection, appropriate parameters and subroutines are chosen and adjusted to perform these functions by micro-controller or by DSP. Briefly speaking, these parameters determine the number of enabled hardware branches, the diversity in transmission, zero padding, the time-domain or frequencydomain spreading, and the spectrum profile.

\section{Programmable OFDM-CDMA receiver}

The same as transmitting, there are six receiving variations should be accommodated for six system scenarios. A softwarebased Rake receiver is illustrated in Fig. 6. Due to the principle of OFDM transmission, the number of sub-carrier is generally selected that signals on each sub-band suffer from frequencynonselective fading. Therefore, only one finger is sufficient for most cases. However, there may be some situations that the fading in sub-bands is hard to be maintained frequencynonselective. Thus, the number of turned-on fingers could be a pre-defined value or is controlled by the result of channel estimation to combat multi-path effect. The delays between fingers are also adjusted according to the estimation result. Fig.7 depicts the programmable structure of the fingers, where eight functions, F1 F8, are defined. The decision of symbol detection could be used for next step synchronization if the precision is high enough. These functions are defined as:

F1: It controls the sample rate of the received signals according to the selected scenario, including the required number of input in the following FFT.

F2: Function 2 determines the ratio of $S / P$ conversion and the number of parallel outputs (PO) according to the selected scenario. F3: Function 3 decides which type of FFT will be executed. It consists of two sub-functions where the first performs circularshift zero padding for MT-CDMA modes and the second determines the operation points of FFT device. General FFT operates in NOC form for MT-CDMA modes and in regular form for MC-CDMA and MC-DS-CDMA modes by disabling the first sub-function. (See Appendix)
F4: Function 4 controls the filtering of samples after FFT to discard the signals out of the sub-bands and determines the enabled tapped-delay-lines. The pass window (PSW) of zero forcing equals the number of sub-carriers used in transmission. F5: Function 5 is executed in the dispreading stage such that each output of F4 is despread according to the spreading type (constant or cyclic type) of the selected scenario by multiplying the corresponding codes. The despreading structure is a tapped-delayline (TDL) form and the number of enabled TDL equals ZFW in F4. F5 determines the number of enabled tap (ET) and the time spacing (TS) of each tap. For MC-CDMA modes, only one tap is needed and each line corresponds to one bit of the spreading codes. For MC-DS-CDMA and MT-CDMA modes, the number of taps in each line equals the spreading factor.

F6: Functions 6 determines the sampling rate (SR) for taking samples on each line at the dispreading stage. The sampling rate equals the OFDM symbol duration.

F7: Based on the collection of samples from each line of all fingers, the detector makes symbol decision by combining these diversities. Different combining methods could be applicable in this stage by DSP.

F8: It controls the de-multiplexing in multi-code mode to reconstruct the original data sequence from the effective streams.
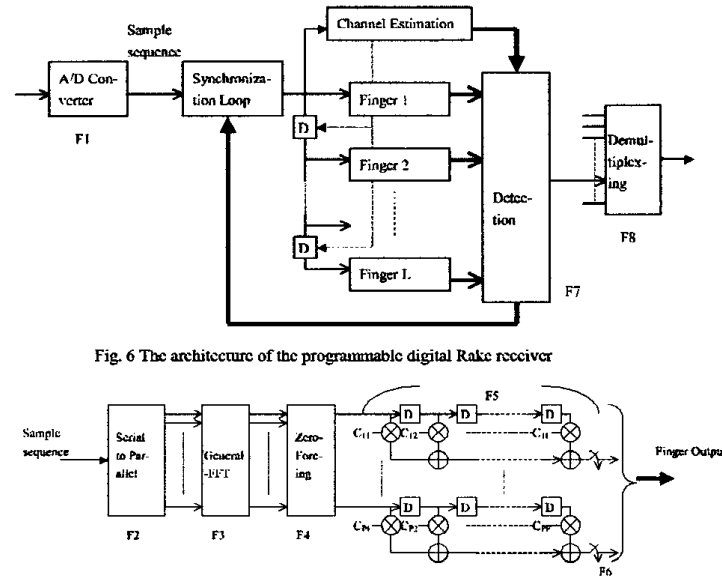

Table 2.

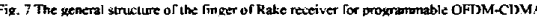

\begin{tabular}{|c|c|c|c|c|c|c|}
\hline & $\begin{array}{c}\text { FI } \\
\text { Sample Rate }\end{array}$ & $\begin{array}{l}\text { F2 } \\
\text { PO }\end{array}$ & $\begin{array}{c}\text { F3 } \\
\text { IFFT }\end{array}$ & $\begin{array}{c}\text { F4 } \\
\text { PSW }\end{array}$ & $\begin{array}{c}\text { F5 } \\
\text { ET; TS }\end{array}$ & $\begin{array}{l}\text { F6 } \\
\text { SR }\end{array}$ \\
\hline$(A, m, M C)$ & $\left.2^{\left[\operatorname{lwg}_{y}(P F)\right.}\right] / P T_{s}$ & $2^{\left[\log _{2}(P F)\right]}$ & Regular & $P F$ & $1 ; 0$ & $1 / P T_{s}$ \\
\hline$(A, m, V S L)$ & $2^{[\log (, P F)]} / P T_{s}$ & $2^{\left[\log _{2} P F\right)}$ & Regular & $P F$ & $1 ; 0$ & $1 / P T_{s}$ \\
\hline$(B, m, M C C, L)$ & $F 2^{\left[\log _{n}+P I \eta\right]} / P T_{s}$ & $2^{\left[\log _{2}\left(x_{2} P\right)\right]}$ & Regular & $P L$ & $F ; P T / F$ & $1 / P T_{s}$ \\
\hline$(B, m, V S L, L)$ & $F 2^{\left[\mathrm{kEZ}_{2}\left(P_{L}\right)\right]_{/ P T_{s}}}$ & $2^{\left\lceil\log _{3}(P L)\right\rceil}$ & Regular & $P L$ & $F / m ; P T_{s} / F$ & $m / P T_{s}$ \\
\hline$(C, m, M C)$ & $F 2^{\left[\log _{2} n\right]^{\prime}} / P T_{s}$ & $2^{\left[\mathrm{los}_{3} / p\right]}$ & NOC & $P$ & $F ; P T, / F$ & $1 / P T_{s}$ \\
\hline$(C, m, V S L)$ & $F 2^{\left[\log _{2} p T_{1}\right.} / P T_{s}$ & $2^{\left[\log _{t} p\right]}$ & $\mathrm{NOC}$ & $P$ & $F / m ; P T_{N} / F$ & $m / P T_{s}$ \\
\hline
\end{tabular}

\section{Implementation Issues}

The software architecture of the proposed programmable multirate OFDM-CDMA transceiver can be realized by the general hardware structure in [4] as an extension. Different scenarios 
have different requirement of bandwidth and sampling rate. Due to the accommodation of the six multi-rate OFDM-CDMA sceuarios, it forces the specification of the D/A converter and the low-pass filter should satisfy all the requirements. Thus, the D/A converter should support input rate higher than any possible sampling rate and the bandwidth of the low-pass filter should accommodate all scenarios. Another practical issue is the peak-toaverage power (PAP) ratio problem, which causes the inefficiency of power amplifiers in RF. The PAP problem exists inherently in OFDM systems and can be reduced by techniques such as signal distortion, error correcting, and scrambling [6]. However, the introducing of multi-rate traffic challenges the power AMP severer. Among these scenarios, multi-code access may cause larger instant power due to its concept of parallel transmission. Therefore, we should select a power AMP whose linear range could accommodate the largest requirement of multi-rate transmission under the aid of PAP reduction mechanisms.

To eliminate the inter-symbol interference and inter-carrie interference, a guard time $T_{g}$, which is larger than the multi-path spread of channels, should be added on each OFDM symbol after $\mathrm{P} / \mathrm{S}$ conversion in the transmitter and the corresponding removing should exist before $F 1$ in the receiver. After cyclic guard time extension, the OFDM symbol duration $T$ will be $P T s+T g$ in $\mathrm{MC}$ CDMA, and $P T s / F+T g$ in MC-DS-CDMA and MT-CDMA. In addition, to improve the performance, some techniques such as forward error control (FEC) and interleaving could be easily added to this structure without any difficulty.

Although our design focuses on multi-rate applications, this architecture is backward compatible to single rate OFDM-CDMA systems by setting $m=1$, to DS/CDMA systems by setting $P=L=1$ in MC-DS-CDMA mode, and to conventional OFDM systems by setting $F=L=1$ in single user case. Due to the occupied bandwidth of each OFDM-CDMA systems is kept fixed for users of any rate in both MC access and VSL access modes, multi-rate applications do not increase the requirement on some hardware devices, such as the sampling rate of $\mathrm{AD}$ and the bandwidth of low-pass filter.

Processing delay is a major challenge in OFDM systems, especially sensitive in real-time applications. In fact, this programmable transceiver supports the possibility that the number of sub-carriers could be adjusted dynamically according to the channel conditions such that the least sub-carriers attain frequency-nonselective fading at each sub-channel. In the same way, delay-sensitive data transmission is realizable by reducing the number of sub-carriers with the aid of more fingers in Rake receiving.

\section{Conclusion}

We developed a transceiver architecture of multi-rate OFDMCDMA systems and showed its programmability such that the general system can operate under different scenarios with a common hardware structure and reconfigure by software implementation. The future work shall be analyzing the performance of each scenario and figuring out its relationship with these adjustable parameters such that optimal resource programming is realizable for future wireless multimedia applications.

\section{References}

[1] T. Ottosson and A. Svensson, "Multi-rate schemes in DS/CDMA systems", Proc. IEEE Vehic. Tech. Conf., pp. 1006-1010, 1995.

[2] K. Fazel, S. Kaiser and M. Schnell, "A flexible and high-performance cellular mobile communication system based on orthogonal multi-carrier SSMA," Wireless Personal Communications, vol. 2, No. 1, pp. 121-144, 1995

[3] Shinsuke Hara, Ramjee Prasad, "Overview of multicarrier CDMA," IEEE Communication Magazine, pp. 126-133, Dec. 1997.

[4] K C. Chen and S.T. Wu, "A Programmable architecture for OFDMCDMA," IEEE Communication Magazine, pp. 76-82, Nov. 1999.

[5] Sourour, E.A.; Nakagawa, M. "Pcrformance of Orthogonal Multicanicr CDMA in a Multi-path Fading Channcl", IEEE Transactionis on Communications, pp. 356-367, March 1996.

[6] R. Van. Nee, and R Prasad, OFDM for wireless multimedia communications, Artech House, 2000

\section{Appendix NOC-IFFT}

For the $k$ th user, the transmitted signals after modulation is $x_{k}(t)=\sum_{p=1}^{P} b_{k p} \sum_{f=1}^{F} c_{k f} \varphi\left(t-f T_{c}\right) k^{j 2 \pi f_{p} t}=\sum_{f=1}^{F} x_{k f}(t), 0 \leq t \leq T,($ A.1 $)$ where $\Delta f \equiv f_{i+1}-f_{i}=1 / P T_{s}$ and the discrete equivalent form of $x_{k r}(t)$ is: for $n=1 \sim N$,

$$
x_{k f}[n]=\sum_{p=1}^{P} d_{k p}[f]^{j 2 \pi((f-1) P+p) h / N},(f-1) T_{c} \leq t \leq f T_{c},
$$

where $d_{k p}[f]=b_{k p} c_{k f}$.

Definition:

$N$ point NOC-IDFT of a sequence $s[i], i=1 \sim N_{s}$ where $N / N_{s}=F \in \mathbf{N}$ is generated $b v$

(1). At lth sub-period, for $l=I, 2, \ldots, F$.

$$
s^{\prime}[i]=\left\{\begin{array}{l}
s\left[i-(l-1) N_{s}\right],(l-1) N_{s}+1 \leq i \leq l N_{s} \\
0, \text { otherwise }
\end{array}\right.
$$

(2). $y_{l}[n]=F_{\text {NoC }}^{-1}\left\{s[1] \cdots s\left[N_{s}\right], N, l\right\}=\sum_{i=(l-1) N_{*}+1}^{L N_{s}} s^{\prime}[i] \exp (j 2 \pi i n / N)$.

where $N_{s}=N / F, n=I \sim N$, and $l=1 \sim F$.

Therefore, let $N=F P$ and it can be easily shown that $x_{k f}[n]=F_{\text {voc }}^{-1}\left\{d_{k 1}[f] \cdots d_{k P}[f] N, l\right\}=F^{-1}\left\{s^{\prime}[1] \cdots s^{\prime}[N] N\right\}$ (A.3) where $F\{; N$ denotes $N$-point regular DFT. Therefore, circularshift zero padding and regular FFT can implement NOC-FFT. Disabling of F4 goes back to regular IFFT.

For receiving in MT-CDMA systems as [5], we define the NOC-

FFT corresponding to NOC-IFFT, Let $r^{\prime}[n], n=1 \sim N$, be the circular-shift zero padding for $r[n]$,

Definition:

$F$ points NOC-DFT of $r[n], n=1 \sim N_{s}$, is

$$
s_{f}[i]=F_{N o C}\left\{r[1] \cdots r\left[N_{s}\right] F, f\right\}=\sum_{n=(f-1) N_{t}+1}^{N_{s}} r[n] \exp (-j 2 \pi i n / N) \text {. }
$$

$i=I \sim N_{s}$, where $N_{s}=N / F \in \mathrm{N}$

By the same principle, it can be shown that circular-shift zero padding and regular FFT can also implement NOC-FFT. Therefore, sampling $r(t)$ with rate $N / F T c$ and taking $F$-point NOC-FFT with period $T$ will get the desired result. = 